\title{
Can we have mathematical understanding of physical phenomena? ? $^{*}$
}

\author{
Gabriel TÂRZIU
}

Received: 22/09/2017

Final version: $14 / 11 / 2017$

BIBLID 0495-4548(2018)33:1p.91-109

DOI: $10.1387 /$ theoria. 18108

ABSTRACT: Can mathematics contribute to our understanding of physical phenomena? One way to try to answer this question is by getting involved in the recent philosophical dispute about the existence of mathematical explanations of physical phenomena. If there is such a thing, given the relation between explanation and understanding, we can say that there is an affirmative answer to our question. But what if we do not agree that mathematics can play an explanatory role in science? Can we still consider that the above question can have an affirmative answer? My main aim here is to give an account that takes mathematics, in some of the cases discussed in the literature, as contributing to our understanding of physical phenomena despite not being explanatory.

Keywords: mathematical explanation; unexplanatory understanding; mathematical understanding.

RESUMEN: ¿Pueden las matemáticas contribuir a nuestra comprensión de los fenómenos físicos? Una forma de intentar responder a esta pregunta es implicándonos en la reciente disputa filosófica sobre la existencia de explicaciones matemáticas de los fenómenos físicos. Si existe tal cosa, dada la relación entre explicación y comprensión, podemos decir que hay una respuesta afirmativa a nuestra pregunta. Pero, ¿y si no estamos de acuerdo en que las matemáticas pueden desempeñar un papel explicativo en la ciencia? ¿Podemos considerar entonces que la pregunta anterior puede tener una respuesta afirmativa? Mi objetivo principal aquí es dar una explicación que considere que las matemáticas, en algunos de los casos discutidos en la literatura, contribuyen a nuestra comprensión de los fenómenos físicos a pesar de no ser explicativa.

Palabras clave: Explicación matemática; comprensión no explicativa; comprensión matemática.

* The idea for this paper was born while I held a Visiting Fellowship offered by The European Philosophy of Science Association (EPSA) at the Centre for Logic and Philosophy of Science at Ghent University. I wish to express my gratitude to EPSA and to the Centre for the opportunity to develop my research. I owe very special thanks to Professor Erik Weber for many helpful discussions and for providing invaluable comments on earlier drafts of this paper. I would also like to thank the anonymous referees of this journal for commenting on an earlier version of this paper. 


\section{Introduction}

The problem of the role of mathematics in scientific explanations attracted a lot of philosophical attention lately. The most interested in this topic are the mathematical realists, who think that it can offer a good starting point for an argument in support of their position, and the nominalists, who are concerned with refuting the realists' arguments, but there are other parties weighing in also (mostly from the general philosophy of science, where this topic is important for the discussion about scientific explanation). After several years of heated debate, the situation is this: despite the fact that the number of those accepting that there are mathematical explanations of physical phenomena (from now on mathematical explanations) is rising, we are still far from a general consensus that there is such a thing; and, despite the fact that the examples are multiplying, there is little agreement, even among the advocates, about which ones are genuine cases of mathematical explanations and which are not. My aim here is to argue for a new position: one that takes mathematics as conveying understanding in a scientific context, without playing an explanatory part. ${ }^{1}$ On the one hand, I agree with people like Melia (2002), Bangu (2008), Daly and Langford (2009), Saatsi (2011), Rizza (2011), and Räz (2013; 2017), that mathematics does not play an explanatory role in science, but, on the other hand, I also agree with people like Steiner (1978), Colyvan (2001), Baker (2005; 2009; 2012), Batterman (2010), Lyon (2012), Lange (2013), and Pincock (2015), that mathematics —in the cases discussed and in similar ones - contributes to our understanding of physical phenomena. There is an apparent tension here. After all, isn't understanding inextricably linked with explanations? So, how can one claim that mathematics can contribute to our understanding of physical phenomena without playing an explanatory role in science? If understanding is indeed inextricably linked with explanations, my project is doomed from the start. That is why I will first say a few words (section 3.) about the possibility of unexplanatory understanding and the demise of the received view of the link between explanation and understanding. With this out of the way, I will develop an account that shows how, in some of the examples discussed in the literature, mathematics contributes to our understanding without being explanatory. (sections 4. and 5.). Before I do all this, I will present three of the most discussed cases of alleged mathematical explanations together with some of the reasons why we should not accept that they are genuine mathematical explanations (section 2.).

\section{The cases}

We can understand the recent philosophical dispute about the existence of mathematical explanations in science as revolving around a few of cases. Typically, the discussion is carried out this way: the advocates of mathematical explanation come up with one or several examples of what intuitively looks as mathematical scientific explanations and argue that

1 It is important to emphasize from the beginning that my aim here is not to argue that there is no such thing as mathematical explanations. What I am trying to do is to provide an account that saves the intuition that mathematics contributes to our understanding of why something is happening in the physical world, in some of the cases of alleged mathematical explanations discussed in the literature. 
mathematics is doing genuine explanatory work in these cases. Their opponents argue that mathematics plays other, unexplanatory, roles in such cases, so it is wrong to consider them as genuine examples of mathematical explanations. Let us take a look at some of the most discussed such cases to see if the balance is tipping in anyone's favour.

\section{THE ANTIPODAL WEATHER PATTERNS}

Let us consider the following situation: at any given time, we can discover on Earth's surface two antipodal points with exactly the same temperature and barometric pressure. Why is that? Why are there such antipodal points? (Colyvan 2001, 49) Colyvan argues that there is no causal story that we can use to answer this question. Such a story would have been helpful if we would have been interested in some particular points, say $p_{1}$ and $p_{2}$, because in that case we could have used a detailed causal history of the weather patterns in the neighbourhood of $p_{1}$ and $p_{2}$ to account for the particular values that they have at time $t_{0}$. Such a treatment would, of course, take only as an accidental match the fact that the two points have the same values for pressure and temperature. If, for some reason, we believe that we are not dealing with an accident, the only hope for an explanation is to appeal to the Borsuk-Ulam theorem. This is a theorem in topology according to which for any continuous real valued function $f: S^{n} \rightarrow R^{n}$ (from a n-sphere into Euclidean n-space) there exists $x \in S^{n}$ such that $f(x)=f(-x)$ (i.e. maps some pair of antipodal points to the same point). For the case $n=2$, this theorem can be interpreted as saying (assuming that the Earth is topologically equivalent to a sphere and that temperature and pressure change continuously across its surface) that there are always on Earth's surface, at a given time, antipodal points with the same temperature and barometric pressure, i.e. exactly our explanandum. So, if there is an intra-mathematical explanation for the Borsuk-Ulam theorem, i.e. an explanatory proof for it, this can be taken to also explain the observation of the antipodal identical weather points. Colyvan considers that there is such a proof and so that we can speak of a mathematical explanation for the existence of such antipodal points on the surface of the Earth.

There are several problems that stand in the way of taking this example as a genuine case of mathematical explanation. First of all, as Baker $(2005,226)$ argues, since stumbling across such points is not very likely and since it is implausible that the meteorologists would look for them if unaware of the mathematical theorem, we are dealing with a prediction and not an explanation. A second point, made also by Baker (2005), is that in this case we do not even seem to be dealing with a phenomenon in need of an explanation. I would add to this one more worry having to do with the proof of the BorsukUlam theorem and its explanatory virtue: what proof of the theorem does Colyvan have in mind and what makes it explanatory? There are several proofs of this theorem and most of them are by contradiction (including the one in the book Colyvan is referencing, i.e. Kosniowski (1980)), but the proofs by contradictions are not always considered to be explanatory. The only reason for why we should take the proof of this theorem as explanatory that I could find is in a footnote in Baker and Colyvan $(2011,327)$ which says that "there is prima facie evidence against the proof in question being unexplanatory: namely, the connection between (the proofs of) the Borsuk-Ulam theorem and other fixed-point theorems (most notably, Brouwer's celebrated fixed-point theorem). These connections seem to give some plausibility to the proof in question being explanatory." Unfortu- 
nately, this remark is obscure and unhelpful. The authors do not give us any clue about what connection they have in mind here and, also, they do not explain why this connection can count as evidence against taking the proof (whatever it may be) of our theory of interest as being unexplanatory.

\section{THE CICADAS}

Baker $(2005 ; 2009 ; 2012)$ considers that there is an example of mathematical explanation that overcomes all the problems that he found to affect Colyvan's cases: the cicadas example. In the case of North American periodical cicadas the explanandum is the prime-numbered length of the life-cycle of these insects (13 or 17 years, depending on the geographical area). In Baker's view, in order to explain this phenomenon, scientists made essential use of the following mathematical theorem: the lowest common multiple of two numbers is maximal when the numbers are coprime. The explanation goes like this: having a life-cycle period which minimizes intersection with other periods is evolutionarily advantageous because it either helps with avoiding predators or it diminishes the chances of hybridization with similar subspecies. But, from the theorem presented above, it follows that the primenumbered periods minimize the intersection with other periods. So, periodic organisms such as the North American cicadas are likely to evolve prime periods.

As it turns out, this case is not problem free either. Davide Rizza (2011) argues, for example, that this explanation can be given without using any mathematical facts because the property of being prime - of central importance in the explanandum - is not, in this case, and should not be interpreted as a property of some mathematical entities, but should be taken as having to do with the properties of time intervals corresponding to life-cycles. Now, suppose Rizza is wrong and the primeness referred to in the explanandum is in fact a mathematical property. What this implies is that the explanandum is not in fact a pure physical phenomenon, but a mixture of physical and mathematical elements (Bangu, 2008). But, then, this case is not a case of mathematical explanation of physical phenomena as Baker would have us think.

A different argument against taking this case as a genuine mathematical explanation of physical phenomena can be found in Daly and Langford (2009). It is argued here that the role of numbers in this and similar cases is only to index a given duration (the durations of the life-cycles of the cicada) and so it plays no explanatory part. If we take this into account, we can give the following mathematics free paraphrase of the explanation: "the reason why the life-cycle of a given species of cicada is of the given particular number of units is that the duration which that number of units picks out minimizes the cicadas' contact with other predatory species that inhabit the cicadas' environment" (657).

\section{THE HONEYCOMB}

A different case that enjoys a great deal of attention in the literature is the one proposed in Lyon and Colyvan (2008): the explanation of the structure of the bee's honeycomb. The idea in this case is that biologists explain why the bee's honeycomb has that particular shape with the help of the following mathematical theorem (the honeycomb theorem): a hexagonal grid is the optimal way to divide into regions of equal area with least total perimeter a Euclidean plane. The explanation goes as follows: in order to win the 
natural selection fight, honeybees had to choose the most economic (in terms of labour and amount of wax used) way to build their honeycombs. As it is clear from the mathematical theorem presented above, from all the possible shapes, the hexagonal grid is the most economical in the relevant respects. This is why the honeycombs have that boggling shape.

Saatsi (2011), Lange (2013) and Räz (2013), among others, found problems with accepting that this case represents a genuine mathematical explanation. Tim Räz argues that, for mathematical reasons, "the explanation proposed by Lyon and Colyvan is an inadequate explanation of the structure of the bee's honeycomb” (Räz 2013, 354). He argues that the honeybees' honeycombs are in a nontrivial way three-dimensional and this makes the explanation at least incomplete; and that, for the same reasons, the honeycomb theorem is probably irrelevant for the explanation of comb structures.

Saatsi (2011) and Lange (2013) both give arguments that apply equally to the cicadas and honeycomb examples. In Saatsi's view, the role of mathematics is that of representing a "crucial physical fact to which the full evolutionary explanation of the phenomenon then appeals" (Saatsi 2011, 146). He argues that, in both the cicadas and the honeycomb examples, the role of mathematics is not explanatory, but representational and knowledge-conferring.

Lange takes both examples to be cases of causal, not mathematical, explanations. Regarding the explanation of the honeycomb structure, he says, for example, that it "works by describing the relevant features of the selection pressures that have historically been felt by honeybees, so it is an ordinary, causal explanation, not distinctively mathematical" (Lange 2013, 501).

As I see it, the arguments against taking the cases presented above as examples of mathematical explanations are pretty convincing. But, in this case, anyone that feels the way I do is left with the following conundrum: why are there so many philosophers that tend to think that mathematics can and does play an explanatory role in cases such as these? There are two opposite directions one can take in trying to answer this question: either argue that these philosophers have wrong intuitions about the role of mathematics in these cases, or argue that their intuition is good but that, for some reason, they draw the wrong conclusions from it. I prefer the latter direction. In my opinion, the advocates of mathematical explanations have a good intuition about the role of mathematics in such cases, i.e. that it plays an important part in our scientific endeavour to understand some physical phenomena. The fact that they are going past this to argue that mathematics is explanatory has to do with the way they see the relation between understanding and explanation: if understanding is taken as inextricably linked with explanation, saying that mathematics can contribute to our scientific understanding of physical phenomena amounts to nothing less than saying that it can play an explanatory role in science.

Now, given the fact that there are good reasons for considering that mathematics does not play an explanatory role in science (in cases such as the ones discussed above), combining the intuition about the contribution of mathematics to our understanding with this view on the relation between understanding and explanation can only make the former 
seem wrong. I do not believe this intuition is wrong, though, so I will try to rescue it. In order to do this, I believe it is important to first say a few words about the relation between understanding and explanation-basically to point to the reasons for not taking them as inextricably linked. After this, I will give an account that shows how in the examples presented above mathematics contributes to our understanding despite not playing an explanatory role.

\section{Explanation and understanding}

What relation is there between explanation and understanding? If we look for an answer to this question in the extensive literature on scientific explanation, we find something along these lines: understanding is inextricably linked with explanation. Despite the fact that there is a widespread disagreement among the philosophers of science about the correct account of scientific explanation, they seem to have reached a consensus regarding its relation with understanding. Peter Achinstein $(1983,16)$, Wesley Salmon (1984, 259), Michael Friedman (1974, 7), James Woodward (2003, 23), Michael Strevens $(2008,3)$, for example, take explanation and understanding to be fundamentally connected.

Fortunately, the view about this relation is (slowly) starting to change. Philosophers such as de Regt, Grimm, Kvanvig, Elgin, Pritchard and many others argue recently that understanding is (separately) worthy of philosophical attention and that its study can contribute new insights to many traditional debates in philosophy. This newfound interest in understanding creates the perfect background against which the possibility of understanding without explanation can be taken seriously. This has already been done in Kvanvig (2009), Lipton (2009), Gijsbers (2013) and Hindriks (2013), among others.

Most recently, the strong link view about the relation between explanation and understanding is defended in Strevens (2013). Strevens takes understanding to be just a matter of being in the right relation with an explanation, i.e. grasping a correct explanation. In his view, grasping is more than just knowing that something is the case. Grasping is a kind of understanding: it is understanding that or direct apprehension, which resembles a sort of conscious realization that something is the case. For example, grasping that the cat is on the mat means being fully aware of the cat, the mat, and the spatial relation between them (Strevens 2013, 511). Grasping an explanation, on the other hand, means understanding that the propositions that compose it have the right structure (e.g. they form a deductive argument for the explanandum) and that the states of affairs represented by them do obtain (idem).

The problem with Strevens's and other similar views about the relationship between understanding and explanation is that it does not rule out the possibility that understanding can be obtained in some other way than just by grasping an explanation. Once we disentangle understanding from explanation (as the philosophers mentioned above are doing), this possibility can no longer be ignored. ${ }^{2}$ In order to rule out unexplanatory understanding, two things need to be shown: (a) that understanding cannot be obtained by grasping other things than explanations, and (b) that understanding always involves grasping. As far as I know, nothing like this can be found in the literature. Instead, what we can find are arguments that challenge this strong link view. Lipton 
(2009) 3 and Gijsbers (2013), for example, offer what can be taken as arguments against our point $(\boldsymbol{b})$, because both of them take understanding not as grasping something, but as a form of knowledge (for Gijsbers it is knowledge of connections between phenomena, while for Lipton it is knowledge of causes, of necessity, of possibility, and of unification) and show how it can be obtained in some other way than through explanations. Lipton takes understanding to be the cognitive benefit provided by explanations and argue that it can also be obtained "by routes that do not pass through explanation" (Lipton 2009,44$)$, e.g. by the use of images and physical models, by manipulation or by internalisation of Kuhnian exemplars. A different view can be found in Gijsbers (2013) which argues that that there is a type of unexplanatory understanding that we can acquire through correct classification of phenomena. In his view, "when we realise which phenomena are like which other phenomena, which phenomena ought to be unified under the same concept and which ought to be kept separate, we advance our understanding" (Gijsbers 2013, 521).

I want to consider a different way to challenge the strong link view, one that takes seriously the possibility that understanding can also be obtained by grasping other things than explanations. Let us take a look at the following visual proof (Fig 1.) by Benjamin Klein and Irl Bivens (Nelsen 1993, 120) of the geometric series $1+r+r^{2}+\cdots=\frac{1}{1-r},(0<r<1)$. I think it is undisputable that most of us are able to understand why this geometric series equals $\frac{1}{1-r}$ just by looking at the diagram presented below. I also consider it to be obvious that, in this case, there is no explanation available, ${ }^{4}$ nor some type of knowledge we can rely on for understanding. The fact that we understand something has to do only with grasping the geometric relationships that hold in the diagram below, most importantly that the triangles PQR and TSP are similar and so that the ratios of their corresponding sides must be the same, i.e. that $\frac{S T}{P S}$ must be the same as $\frac{P Q}{R Q}$. Since the idea in this section is only to show that unexplanatory understanding is possible, it would take us too far afield to enter into details about the account that would best accommodate this type of cases. For what is worth, my view is that Lipton's $(2009,45)$ remarks about 'the use of images and physical models' is a good starting point for developing such an account (provided we find a way to dissociate it from Lipton's knowledge account of understanding).

There are two lessons to be learned from discussing this case. First of all that there is more to understanding than just grasping explanatory relations, and secondly that we can have unexplanatory understanding in mathematics as well.

2 A similar point is made in Lipton (2009).

3 Strevens (2013) discusses Lipton's paper, but, as I see it, his attempt to dismiss Lipton's view is flawed because Lipton's aim is not, as Strevens claims, to give "good reasons to think that understanding can come by grasping facts that are not explanatory" (Strevens 2013, 5014, emphasis added).

4 For those unconvinced, I cannot do better here than to refer to Lipton's (2009) discussion about the possibility of tacit explanations. 


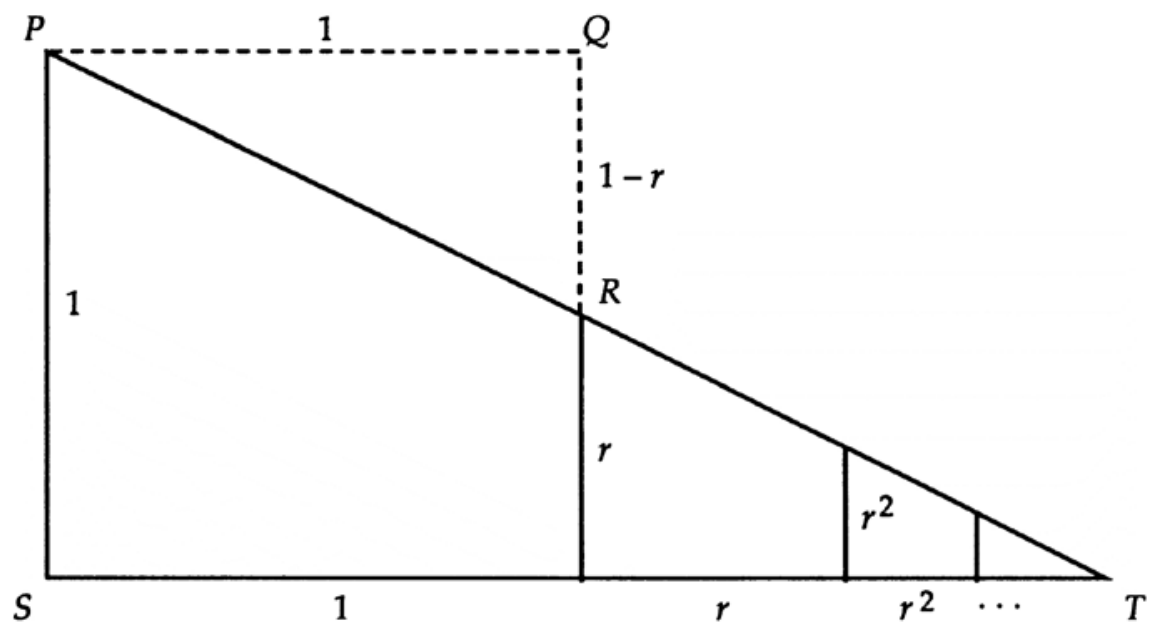

Figure 1

\section{A new account}

Until now I tried to do two things. I presented three of the most discussed examples in the literature and showed what reasons there are for being reluctant in accepting that they represent genuine cases of mathematical explanation. I take these reasons to be pretty convincing, but I also consider that there is something right about the intuition that mathematics does contribute to our understanding in these cases. But, is understanding without explanation even possible? If not, then it makes no sense to entertain such an idea. This brings us to the second thing I tried to do here, namely show that unexplanatory understanding is far from being an absurdity. It is time now to put things together. In what follows I will try to give an account that can make sense of the idea that mathematics, even though does not play an explanatory role, contributes to our understanding in such cases. I believe that we can obtain this by altering Mark Steiner's account of mathematical explanation. There are two ways to do this: either by extending it so as to allow the transfer (beside of explanations) of unexplanatory understanding, or by narrowing it so as to take only understanding as transferable. I prefer the latter because I consider Baker's (2012) criticism (see below) to be correct and I believe that, unlike understanding, explanations cannot be transferred. But someone who isn't persuaded either by Baker, or by what I am about to say next, but is convinced by the arguments against taking the cases discussed in section 2 . as examples of mathematical explanations, can still find what I am about to say next (section 5. in particular) interesting if she ignores the discussion about the transfer of explanations and opts for the former type of altering of Steiner's account. ${ }^{5}$

5 It is important to emphasize at this point the fact that, even though I argue below that explanations are not transferable, my account does not depend on this being the case. All that it needs to work is the existence of unexplanatory mathematical understanding and the possibility of transferring understanding between different domains. 
For Steiner, a characteristically mathematical explanation of a physical phenomenon is one in which, if "we remove the physics, we remain with a mathematical explanation of a mathematical truth" (Steiner 1978, 19). The main idea here, even though not explicitly stated by Steiner, seems to be that explanatory power somehow trickles from the purely mathematical explanation into the mathematical explanation of the physical phenomenon. Suppose we have, for example, two domains $\mathrm{D}_{1}$ and $\mathrm{D}_{2}$ and two facts, $f_{1} \in \mathrm{D}_{1}$ and $f_{2} \in \mathrm{D}_{2}$. Suppose further that we have an explanation for $f_{1}$ and we lack an explanation for why $f_{2}$ is the case, but that there is a mapping relation linking $f_{1}$ and $f_{2}$. Now, Steiner's thesis is that, given this mapping, we can use the explanation for $f_{1}$ to also account for why $f_{2}$ is the case. There seem to be big problems, though, with such a view. Alan Baker dedicated a whole paper to the task of bringing them to light (Baker, 2012). He argues that the examples of mathematical explanations discussed in the literature fail (what he calls) the Steiner Test ${ }^{6}$ in two respects (Baker 2009, 623). In some of these examples, there is a proof for the mathematical theorems used, but this proof is not explanatory; so, there is no way that these explanations can inherit something from the pure mathematical counterpart. In other examples we do not even have the luxury of debating if the proof of the mathematical result used is explanatory or not because there is no such proof to begin with. So, in such examples, the explanatory contribution of the mathematical part cannot be traced back to something coming from pure mathematics.

I agree with Baker's points, but I believe that there is something even more problematic with Steiner's account. As I said earlier, its main idea is that explanations can be transferred from one domain to another. How is this possible? I could not find in the literature any explanation. The most we can find is that, intuitively, such a transfer makes sense. For example, in Baker and Colyvan (2011) it is claimed that "Maintaining that there are intramathematical explanations but that these explanations never permeate beyond the boundaries of mathematics is prima facie implausible" (327). I don't find this implausible at all. Actually, I believe that there is something that can make us look with suspicion at this idea. It involves the following intuitive principle: ${ }^{7}$ things happen in a certain system (i.e. a set of interrelated things) only because of the facts that have to do with that system or its relations with other systems. But, if this is indeed the case, it means that any explanation of something happening in a certain system has to be given in terms of facts about that system, i.e. of the laws governing it, of the relations holding between its parts etc. Someone adhering to this principle would no doubt find it strange to think that one's failure to distribute evenly five stamps to two people has something to do with the mathematical fact that five cannot be divided evenly by two, rather than with some physical properties of the stamps (e.g. that splitting a stamp in two destroys it). ${ }^{8}$

This might not be convincing enough, though, but that is ok, as it was meant only as an attempt to resist the appeal to plausibility made in Baker and Colyvan (2011) by showing that someone can have different intuitions. I believe, though, that we can go beyond a

6 Roughly, this test can be formulated this way: for a scientific explanation to be genuinely mathematical, the following has to be the case: if we remove the physical cover, we should be able to find a pure mathematical explanation of a mathematical fact.

7 Of course, this may not seem so intuitive to all, but it is ok since I believe that the same happens with the claim made in Baker and Colyvan.

8 See Lange $(2013,488)$ for a different opinion. 
simple exchange of intuitions towards settling this matter by taking a closer look at the way mathematics is applied in science. According to a popular view, what makes the applicability of mathematics possible is the existence of a structural similarity between it and some portion of the physical world. This idea is best captured in Christopher Pincock's mapping (or structuralist) account of applicability. According to Pincock (2004a, 2004b) the best way to understand applicability is in terms of a mapping with certain structural properties between a mathematical domain and a physical situation - "for each mapping involved in an application, the existence of the mapping will depend only on a series of mathematical relations obtaining in the relevant mathematical domain (and, of course, on various physical relations obtaining in the physical situation)" (Pincock 2004a, 70). For example, in the case of distributing evenly five stamps to two people, we have a mapping from stamps and people to a segment of the natural numbers and we interpret the process of distributing stamps to people as the mathematical operation of divisibility. With this in place, we proceed by substitute reasoning (i.e. model based reasoning) to determining if the task is accomplishable. Since five cannot be divided evenly by two, given the mapping between this situation and elementary arithmetic, we conclude that the task is impossible. There is no explanation here. We simply use substitute reasoning to determine if something is or is not the case in the physical situation. ${ }^{9}$ I believe that what generates the impression that something different is going on here is the fact that we are dealing with a why-question and the false belief that this automatically places us in an explanatory context, i.e. the answer is/needs to be an explanation. But not every answer to a why-question is/needs to be an explanation.

Let us consider a different case: the relationship between a city and its map. Does it make sense to think that, sometimes, when we use a map to figure out something about a city, we are dealing with a transfer of explanations? We can be under the impression that this is exactly what we are doing, because of misleading cases like the following one: a tourist asks you if she can get to some touristic attraction in the city by taking a certain route. You explain to her why that is impossible by showing on the map that that route leads in an opposite direction. So, it seems, you used the map to explain why something is the case in the city. This false impression is based on a confusion of explaining something with correcting a false opinion about something. As in the case discussed above, the confusion is generated by the 'why' involving context. This makes us think that we are in an explanatory context, even though the answer in not actually an explanation. In fact you are not explaining anything to the tourist, but use the map to impart knowledge about the city. ${ }^{10}$

Until now we discussed two cases in which we have a 'why' involving context, but no explanation, so transfer is out of the question. What about those situations in which we actually have an explanation? Let's use the last case for considering this possibility. Suppose we have an explanation for why something is the case on the map (e.g. why if you just visited the Louvre and you want to go next to Notre Dame and the Arc de Triomphe it is better to head first to Notre Dame). Can it be transferred to make sense of some city related fact? I believe that the best answer to this question can be found in Bueno and Coly-

9 We rely on our "belief that the 'game' of elementary arithmetic provides a good model" (Leng 2002, 413) for some of our practices to learn something about a particular situation.

10 According to the mapping account, the relation between applied mathematics and the physical world is not very different from the relation between a map and a city, so it is not far-fetched to think that something similar also happens with applied mathematics. 
van (2011). Here it is claimed that: "it would be very odd to think of the map as providing an explanation of any facts about the city. At least, it would be odd to think of the street map offering an explanation that wasn't just standing proxy for another physically-significant explanation" (p. 351). The role of the map is not to provide transferable explanations, but to make obvious certain facts about the city which can then be used to construct (map non-involving) explanations. Bueno and Colyvan do not take this as an argument against the transferability of explanations, though, but as showing that the mapping account cannot accommodate such a thing and so that it doesn't properly address an important aspect of applied mathematics (i.e. its explanatory role in science). "According to the mapping account, mathematics is merely a representational tool, and any explanations that drop out of the mathematics must be just standing proxy for the real physical explanation" (p. 351, footnote 11). Supposedly, this problem is overcome by their inferential conception of applied mathematics. On Bueno's and Colyvan's account, "the fundamental role of applied mathematics is inferential: by embedding certain features of the empirical world into a mathematical structure, it is possible to obtain inferences that would otherwise be extraordinarily hard (if not impossible) to obtain" (Bueno and Colyvan 2011, 352). The main point behind the inferential conception is that mathematics is not just a representational system like a map because it comes equipped with a powerful inferential structure and it is this that enables it to be so useful in science. From this perspective, mathematics can play an explanatory role in science because its inferential structure allows the formulation of explanatory relations that, if a suitable mapping is in place, can be carried to the physical domain (Bueno and Colyvan 2011, 353,365). As I see it, there is an important problem with Bueno's and Colyvan's solution to the problem of transfer. A crucial presupposition in this account is that we are dealing with certain mappings between the two domains (p. 353). This is important because, as it transpires from the discussion above, the problem with transferring map involving explanations is not that the city maps are not complex enough to allow the formulation of explanations, but it has to do with the fact that, whatever map involving explanations we have, they can only act as proxy for explanations in terms of city facts. So, the problem is generated by the type of relation that holds between the map and the mapped domain. But, if this is the case, the inferential account doesn't fare better in accommodating transferred explanations than the mapping account. No matter how complex the system used to map something is, it is still a representational system (if used in this way) and so it inherits whatever problems such systems have with the transfer of explanations. Of course, unlike Bueno and Colyvan, I don't consider this as proof that some account of the applicability of mathematics is flawed, but as evidence that explanations cannot be transferred.

I tried to show until now that, pace Steiner, explanations are not transferable. I didn't offer an impossibility argument, though. Instead, my strategy was to show that, if we take a closer look at the role of mathematics in applied contexts and at the relation between it and the physical world, we obtain a clearer picture about the way mathematics can help us solve empirical problems and extend our knowledge about the physical world, and about the type of confusions that may lead one to think that explanations actually trickle from one domain to another. In the first two cases, the confusion was generated by the 'why' involving (apparently explanation related) contexts. In the last case, complexity was the issue: if a representational system is very complex, it can make us think it has special virtues like leaking explanations. 
What about understanding? Can it be transferred from one domain to another? Let us consider the following case: we have two non-interacting but analogue systems $\mathbf{A}$ and B, i.e. between them there can be a mapping relation based on similarity of relations. Suppose further that in $\mathbf{A}$ we have understanding (obtained in no matter what way) of the relation $\mathbf{R}$ holding between some fact $x$ and some other fact $y$; but in $\mathbf{B}$ (for whatever reason) we have no such understanding of some corresponding relation. Can we transfer the understanding we have in $\mathbf{A}$ to $\mathbf{B}$ ? Yes, we can. Unlike in the case of explanations, here we are not dealing with a change of subject when we change systems. If the mapping between $\mathbf{A}$ and $\mathbf{B}$ preserves all the structural relations, then $\mathbf{R}_{x y}$ and $\boldsymbol{R}_{\boldsymbol{x}} \boldsymbol{B}_{\boldsymbol{y}} \boldsymbol{B}$ are the same in all important aspects. So, if we understand something about one, we automatically understand the same thing about the other. If, for example, we have a map based understanding of the relation between the relative positioning of some touristic attractions, we automatically acquire the same understanding in city related terms. ${ }^{11}$ Similarly, knowing that, when traveling through a wire, electricity exhibits a similar behaviour to that of a liquid allows us to transfer whatever understanding we have of Poiseuille's law to making sense of Ohm's law. Unlike in the case of explanations, it doesn't make sense to say that the map/liquid based understanding stands proxy for a city-facts/electricity-facts involving understanding, because understanding has to do with grasping relations (Kvanvig 2009,96) and there is no noteworthy difference between the relevant relations from the two domains.

So, in my view, we can speak about mathematical understanding of physical phenomena just in those cases in which (to paraphrase Steiner) if we remove the physics we remain with the understanding of a mathematical fact.

\section{The cases, again}

If what I said until now is correct, then what we are dealing with in the cases presented in section 2 . is something like this: we have an intra-mathematical understanding for why a certain mathematical result is the case and, because of the structural similarity between it and some physical system, we can use this understanding (by transferring it) to make sense of something that is the case in the physical world. This way mathematics is relevant in such cases without being explanatory.

Let us consider the meteorological example. Even though mathematics is not explanatory in this case, it is nonetheless relevant for our understanding of the possibility of there being on the surface of our planet, at any time, two antipodal points with exactly the same temperature and barometric pressure. It is so because there is a structural similarity between the Earth and a three dimensional Euclidean sphere and because temperature and barometric pressure are continuous quantities. Given this, we can translate the two-dimensional case of the Borsuk-Ulam theorem into meteorological terms and so we can use whatever (intra-mathematical) understanding we have about it to make sense of the ana-

${ }^{11}$ It is important to acknowledge at this point the limitations of the understanding obtained through transfer: a map based understanding of something about e.g. Paris won't compare with the understanding acquired through direct city experience. It won't help us, for example, understand why almost everybody loves this city. There is only so much that a mapping can do to foster our understanding. 
logue physical situation. We can better grasp why this is the case if we look at the simpler one-dimensional version of this theorem: for any continuous real valued function $f$ on a circle there is a point $x$ on the circle such that $f$ takes the same value at $x$ and at its antipode. This version can be proved with the help of Bolzano's theorem ${ }^{12}$ in the following way: let $g$ be a continuous real valued function on a circle, and $g(x)=f(x)-f(-x)$. If $g(x)=0$, then $f(x)=f(-x)$, so we have our points. Otherwise $g(x) \neq 0$, so either $\mathrm{f}(x)>f(-x)$, or $f(x)<f(-x)$. Suppose $f(x)>f(-x)$. Since we are dealing with a circle, we can go around so that $f(x)<f(-x)$. But then, by the Bolzano's theorem, there must be a point $y$ between $x$ and $-x$ such that $g(y)=0$, i.e. at which $f(y)=f(-y)$. So, what we have here is this: we understand that the Borsuk-Ulam theorem is the case because Bolzano's theorem is the case. But, since the Earth's equator is structurally similar to a circle and temperature is a continuous quantity, we can also use Bolzano's theorem to make sense of the following situation: at any time, there are on the Earth's equator two antipodal points with exactly the same temperature. We can proceed this way. We start with two antipodal points $T_{1}$ and $T_{2}$ on the equator and we assume that the air temperature at $T_{1}$ is higher than at $T_{2}$, so that the difference between them is greater than zero. Suppose now that we move these points around the equator so that $T_{2}$ arrives in the original place of $T_{1}$ and vice versa. In this case, the air temperature at $T_{2}$ would increase gradually until it will be higher than that at $T_{1}$, where we will have a gradual decrease in temperature-so, the difference between them would be smaller than zero. But then, by Bolzano's theorem, somewhere in between these places, the difference in temperature between $\mathrm{T}_{1}$ and $\mathrm{T}_{2}$ must have been zero, i.e. $\mathrm{T}_{1}$ and $\mathrm{T}_{2}$ must have had the same temperature.

In the case discussed above, we understand something about the physical world with the help of mathematics because there is a structural similarity between the thing we lack understanding of and some mathematical fact that we understand. There are situations, though, in which mathematics is indirectly helpful for understanding; for example, when we need help with finding an explanation for a particular phenomenon. I consider this to be the case with the two examples from biology presented in section 2 . In these cases we are dealing with optimality explanations (Baron 2013; Baker 2016), i.e. explanations that work by revealing those factors that affected adaptation in a particular case. Mathematics plays an important role here in helping us find/construct optimality explanations for the phenomena of interest.

The main idea behind evolutionary optimality explanations is that, by favouring those traits and that behaviour that maximizes an organism's fitness, natural selection acts as an optimizing agent. These explanations usually proceed this way: given natural selection and a set of constraints (historical, environmental, developmental), a certain organism has evolved specific traits or behaviour (foraging, sexual, migratory etc.) because having those traits or displaying that behaviour maximizes the value of some fitness related parameter, e.g. dung flies copulate on average for 36 minutes because this is the best strategy for maximizing the average number of eggs fertilized (Parker 1978). The problem is that, on its own, this type of explanation is not very compelling-someone may doubt that a strategy is indeed optimal and/or may be puzzled by the fact that it is that way (e.g. is a copulation

12 According to this theorem, if $f$ is a continuous real valued function on a closed interval $[a, b]$, and $f(a)<0<f(b)$, then there is a point $c \in(a, b)$ such that $f(c)=0$. 
of 36 minutes indeed optimal for dung flies? If it is, what exactly makes it that way?). So, in order to give it more substance, the optimality theorists need to provide evidence that a particular strategy is indeed optimal in a particular selective context and elaborate on what makes it that way. To do this, they construct a model ${ }^{13}$ in which all the relevant elements (or, at least, those considered to be that way) of the adaptive situation are taken into consideration for determining the fitness consequences of adopting every strategy from a set of plausible alternatives. In such a model, fitness is expressed as a function of some variables. This is where mathematics plays a crucial part by helping with determining what combination of variable values maximizes fitness - in those cases in which what is optimum for an individual is not affected by what others are doing, we need only the technique of differentiation, but when we need to take into account the behaviour of other individuals, we need to appeal to game theory. In the case of dung flies, for example, fitness can be expressed as a function of $t$ the time spent copulating, $\mu$ the average time taken by the searching male to encounter a female plus the guarding time it spends after the copulation, and $\mathrm{F}(\mu, t)$ the total foraging time needed to replenish the energy and sperm spent during times $\mu$ and $t$. In order to find out the optimal time (i.e. the time that maximizes the fertilization gains of a male $\mathrm{G}(t)$ ), we need to solve the following equation: ${ }^{14}$

$$
\frac{\partial}{\partial t}\left[\frac{G(t)}{\mu+t+F(\mu, t)}\right]=0
$$

If the model predicts that the strategy that provides the best payoffs is close to that observed in nature, then it is safe to say that the model is accurate, i.e. that it makes the correct assumptions about the elements that constrained adaptation in that particular case (Parker and Maynard Smith 1990, 27).

In the honeycomb example, the optimality explanation for why honeybees evolved to produce hexagonally structured honeycombs is that, given natural selection and the particular set of elements that constrained adaptation in honeybees' case, that is the optimal way to build that particular type of structure. The entire explanatory job here is done by natural selection's power to act as an optimization agent and the special adaptation constraining factors that affected the honeybees. I agree here with Lange's contention that what we are dealing with in this case is just an ordinary causal explanation that works by deriving its explanatory power in the same way as any other selectionist explanation (Lange 2013, 500). There is an important question that needs answering, though: what is the role of mathematics in this explanation? Lange takes it to help with describing the explanandum's causal history, but there are some, like Sam Baron, who consider that without "the honeycomb theorem, there is no good reason why honeybees should have evolved to produce the honeycomb structure that they do"15 (Baron 2013, 4). I disagree with both. As I see it, mathematics plays a more important role here than just describing the explanandum's causal history, but a much less important one than acting as one of the elements that constrained adaptation.

${ }^{13}$ For details about how such a model is constructed, see Parker and Maynard Smith (1990).

14 Parker $(1992,1239)$.

15 I interpret this as placing mathematics between the elements that contributed to the way adaptation took place in the honeybees' case. 
Before entering into more details, I believe it is important to look a little bit closer at the difference between this case and the ones discussed above. In those cases, we understand something about the physical world with the help of mathematics because we have a structural similarity between the thing we lack understanding of and a mathematical result. Someone can be under the impression that we have the same thing here. The biological optimization problems can be understood in terms of finding out the strategy $x$ that, given a set of constraints, maximizes a chosen optimization criterion $O .{ }^{16}$ This usually depends on the relationship between the benefits gained from adopting that strategy $\left(B_{(x)}\right)$ and the costs paid for adopting it $\left(C_{(x)}\right)$. If the optimization criterion is taken to be efficiency, as in the case of honeybees, then the best strategy is the one that maximizes the ratio between benefits and costs, i.e. between the amount of honey that can be stored in the honeycomb and the amount of wax used to build it: $O_{x}=\frac{B_{x}}{C_{x}}$. But we can find in mathematics a similar optimization problem, namely that of determining the optimal way (in terms of minimizing the total perimeter) to divide a plane into regions of equal area. If we take $B$ to stand for area, $C$ for perimeter and $x$ for the strategy that maximizes the ratio of $B$ to $C$, then this problem looks like this: for what $x, O_{x}$ is maximal? This problem has been solved recently by Thomas C. Hales. According to Hales (2001, abstract), "any partition of the plane into regions of equal area has perimeter at least that of the regular hexagonal honeycomb tiling."

Someone can consider that these two problems are structurally similar and so that we can transfer whatever understanding we acquire from the mathematical treatment to make sense of the biological conundrum. Things are far from being that simple, though. The most important thing that we need to understand is that there is more to the optimality approach than optimization theory, i.e. the mathematical tool used to figure out what combination of variable values maximize a particular optimization criterion. If we do this, it becomes clear that the above reasoning is based on a faulty view about the optimality theory. The aim of this approach to evolutionary adaptation is not of trying to determine that ${ }^{17}$ or understand why some trait or behaviour is optimal (we assume that from the start by taking natural selection to act as an optimization agent), neither that of finding out what strategy maximizes some criterion, ${ }^{18}$ but about discovering the forces that shaped adapta-

16 Such an interpretation can be supported by something like the following view on optimality explanations: "An optimization explanation is an explanation of some physical phenomenon, $\mathrm{P}$, that proceeds by situating $\mathrm{P}$ as the solution to an optimization problem. An optimization problem is one which seeks to optimize some target feature, $\mathrm{T}$, given certain constraints, $\mathrm{C} 1, \ldots, \mathrm{Cm}$, and certain options, $\mathrm{O} 1, \ldots$, On” (Baker 2016, 3). As it is clear, I hope, from the discussion above, I adopt here a different view on optimality explanations, i.e. the one found in Maynard Smith (1978), and Parker and Maynard Smith (1990).

17 From the perspective of this approach, it does not make sense, for example, to say that honeybees build suboptimal structures (cf. Fejes Tóth 1964 and Tim Räz 2016). If an optimality model's prediction does not fit with what is observed in nature, we do not take the trait or the behaviour of the organism to be suboptimal, but we reject the model as inappropriate. "The essential point is that in testing a model we are not testing the general proposition that nature optimizes, but the specific hypotheses about constraints, optimization criteria, and heredity" (Maynard Smith 1978, 35).

18 Of course, part of the job of the optimization theorist is to try to determine what this strategy is, given a set of constraints, but not as an end in itself. 
tion in a particular case, i.e. about understanding "specific examples of adaptation, in terms of the selective forces and historical and developmental constraints operating" (Parker and Maynard Smith 1990, 27). But mathematics cannot provide this type of understanding. By finding the solution to a mathematical optimization problem, we do not automatically find out why an organism behaves in a particular way, e.g. the mathematical solution to the honeycomb problem does not tell us why honeybees build hexagonally shaped honeycombs. This should be clear from the fact that, depending on what optimization criterion is chosen in a particular case, we can have several mathematical optima. In the case of a foraging organism, for example, there can be several optimization criteria: time minimization, maximization of energetic gain per unit of energy spent, maximization of net energy extraction from the food source, etc. The models based on these will give (usually) different predictions about what is optimal which can make a lot of mathematical sense but can be as far as possible from what is going on in nature. So, what is mathematically optimal is not necessarily biologically optimal.

Let's return now to our main problem: how does mathematics contribute to our understanding in the context of optimality models? It usually doesn't. Take, for example, the case of the dung flies discussed above. Mathematics was important there for finding out the optimal copula duration. If we solve the above equation for determining the optimal time, we obtain the following value for $\left(t^{*}\right):{ }^{19}$

$$
\frac{\partial G\left(t^{*}\right)}{\partial t}=\left[1+\frac{\partial F\left(\mu, t^{*}\right)}{\partial t}\right]\left[\frac{G\left(t^{*}\right)}{\mu+t^{*}+F\left(\mu, t^{*}\right)}\right]
$$

Does this make the case more intelligible? Hardly so. We use mathematics here only as a tool for drawing the implications from expressing fitness as a function of $\mu, t$ and $\mathrm{F}(\mu$, $t)$. There are situations, though, in which mathematics plays a more important role. This is, for example, the case with the honeybees' honeycombs. In this case we have an understanding in mathematics ${ }^{20}$ for why a certain type of structure is optimal and, since a similar structure is found in nature, we use this to search for an explanation for the physical conundrum. Compared with the way we usually proceed when we search for an optimality explanation for a particular phenomenon (see above), here we are going backwards: instead of constructing a model and using mathematics to deduce the optimal solution, we start from what, unrelated to any physical considerations, is considered as mathematically optimal and we try to find an optimality model that is compatible with such a mathematical result. ${ }^{21}$ As with the other optimality explanations, the starting point here is a biological problem (why do honeybees build their honeycombs as hexagonal grids?), but what comes next does not depend on our assumptions about what natural selection maximizes in this particular case, what are the plausible strategies, and what are the fitness consequences of

19 Parker (1992, 1239).

20 As the discussion in section 3. suggests, I do not take mathematical understanding to depend on the existence of an explanatory mathematical proof.

21 My reconstruction of the process by which we arrive at an explanation in these cases (i.e. honeycomb and cicada) is based mainly on the way the cicada case is dealt with in the following papers: Cox and Carlton (1988), Yoshimura (1997), and Goles, Schulz and Markus (2001). 
the different strategies. What comes next is guided by a mathematical optimization result (the hexagonal grid maximizes the area to perimeter ratio). We use our mathematical understanding of this result to search for an optimization criterion in relation to which this result can be taken to reveal the optimal strategy (we can interpret this result as establishing that the hexagonal structure is the best way to produce the largest cells with the minimal expenditure of energy in producing cell wall material. This suggests that a plausible optimization criterion in this case has to do with the energetic demands that honeybees face when constructing their food storages). If such a criterion is found, we have all we need to put together an optimality explanation for the phenomenon of interest.

The same situation can be found in the case of Magicicada. The mathematical result whose understanding guided the search for an explanation in this case is this: prime numbers maximize the lowest common multiple with all lower numbers. This can be taken to suggest that, in this case, what has been affected by natural selection is the intersection between cicadas and other periodical organisms. Then, a plausible optimization criterion for this case would concern time, viz. the frequency of overlap with other periodical organisms - the optimal strategy is, then, the one that maximizes the time between intersections.

\section{Conclusions}

It is generally acknowledged that mathematics plays a remarkably fruitful role in science, but how helpful can it be when it comes to our scientific quest for understanding the physical world? Can mathematics contribute to our understanding of physical phenomena? Some philosophers offer what can be taken as good grounds for an affirmative answer; they argue that we can find in science examples of explanations in which the mathematical part is doing a genuinely explanatory job. But, as others argue, there are powerful reasons for not taking such examples as cases of mathematical explanations of physical phenomena. The problem with this last position is that it fails to account for the intuition that, in the examples discussed, the mathematical part contributes somehow to the understanding of the physical fact in question. My aim in this paper was to offer a way out of this situation by giving an account that takes mathematics as conveying understanding in such contexts even though it is not explanatory. My position is in an important respect similar to Mark Steiner's (1978) account. I believe that there is something that 'trickles' from the purely mathematical context to the physical one, but it is not explanatory power. What gets transmitted is, in my view, understanding.

\section{REFERENCES}

Achinstein, P. 1983. The Nature of Explanation. New York: Oxford University Press.

Baker, A. 2005. Are There Genuine Mathematical Explanations of Physical Phenomena? Mind 114: 223-238.

Baker, A. 2009. Mathematical Explanation in Science. British Journal for the Philosophy of Science 60: 611-633.

Baker, A. 2012. Science-Driven Mathematical Explanation. Mind 121: 243-267. 
Baker, A. 2016. Parsimony and Inference to the Best Mathematical Explanation. Synthese 193: 333-350.

Baker, A., and Colyvan, M. 2011. Indexing and Mathematical Explanation. Philosophia Mathematica 19: 323-334.

Bangu, S. 2008. Inference to the Best Explanation and Mathematical Realism. Synthese 160: 13-20.

Baron, S. 2013. Optimisation and Mathematical Explanation: Doing the Lévy Walk. Synthese 3: 1-21.

Batterman, R. 2010. On the Explanatory Role of Mathematics in Empirical Science. British Journal for the Philosophy of Science 61: 1-25.

Bueno, O., and Colyvan, M. 2011. An Inferential Conception of the Application of Mathematics. Noûs 45: 345-374.

Colyvan, M. 2001. The Indispensability of Mathematics. Oxford/New York: Oxford University Press.

Cox, R. T. and Carlton, C. E. 1988. Paleoclimatic Influences in the Evolution of Periodical Cicadas. American Midland Naturalist 120: 183-193.

Daly, C., and Langford, S. 2009. Mathematical Explanation and Indispensability Arguments. Philosophical Quarterly 59: 641-658.

Friedman, M. 1974. Explanation and Scientific Understanding. Journal of Philosophy 71: 5-19.

Gijsbers, V. 2013. Understanding, Explanation, and Unification. Studies in History and Philosophy of Science Part $A$ 44: 516-522.

Goles, E., Schulz, O., and Markus, M. 2001. Prime Number Selection of Cycles in a Predator-Prey Model. Complexity 6: 33-8.

Hales, T. C. 2001. The Honeycomb Conjecture. Discrete \& Computational Geometry 25: 1-22.

Hindriks, F. 2013. Explanation, Understanding, and Unrealistic Models. Studies in History and Philosophy of Science Part A 44: 523-531.

Kosniowski, C. 1980. A First Course in Algebraic Topology. Cambridge-New York: Cambridge University Press.

Kvanvig, J. 2009. The Value of Understanding, in A. Haddock, A. Millar, D. Pritchard, eds., Epistemic Value, pp. 95-112. Oxford: Oxford University Press.

Lange, M. 2013. What Makes a Scientific Explanation Distinctively Mathematical? British Journal for the Philosophy of Science 64: 485-511.

Leng, M. 2002. What's Wrong with Indispensability? Synthese 131: 395-417.

Lipton, P. 2009. Understanding without explanation, in H. W. de Regt, S. Leonelli, K. Eigner, eds., Scientific understanding: Philosophical perspectives, pp. 43-63. Pittsburgh: University of Pittsburgh Press.

Lyon, A., and Colyvan, M. 2008. The Explanatory Power of Phase Spaces. Philosophia Mathematica 16: 227243.

Lyon, A. 2012. Mathematical Explanations of Empirical Facts, and Mathematical Realism. Australasian Journal of Philosophy 90: 559-578.

Maynard Smith, J. 1978. Optimization Theory in Evolution. Annual Review of Ecology and Systematics 9: 3156.

Melia, J. 2002. Response to Colyvan. Mind 111:75-80.

Nelsen, R. 1993. Proofs without Words: Exercises in Visual Thinking. Washington, D.C.: Mathematical Association of America.

Parker, G.A. 1978. Searching for Mates, in J. Krebs, N. Davies, eds., Behavioural Ecology: An Evolutionary Approach, pp. 214-244. Oxford: Blackwell Scientific Publications.

Parker, G.A. 1992. Marginal Value Theorem with Exploitation Time Costs: Diet, Sperm Reserves, and Optimal Copula Duration in Dung Flies. The American Naturalist 139: 1237-1256.

Parker, G. A., and Maynard Smith, J. 1990. Optimality theory in evolutionary biology. Nature 348: 27-33.

Pincock, C. 2004a. A Revealing Flaw in Colyvan's Indispensability Argument. Philosophy of Science 71: 6179.

Pincock, C. 2004b. A New Perspective on the Problem of Applying Mathematics. Philosophia Mathematica 12: $135-161$.

Pincock, C. 2015. Abstract Explanations in Science. British Journal for the Philosophy of Science 66: 857-882. 
Räz, T. 2013. On the Application of the Honeycomb Conjecture to the Bee's Honeycomb. Philosophia Mathematica 21:351-360.

Räz, T. 2017. The Silent Hexagon: Explaining Comb Structures. Synthese 194: 1703-1724.

Rizza, D. 2011. Magicicada, Mathematical Explanation and Mathematical Realism. Erkenntnis 74: 101-114.

Saatsi, J. 2011. The Enhanced Indispensability Argument: Representational Versus Explanatory Role of Mathematics in Science. British Journal for the Philosophy of Science 62: 143-154.

Salmon, W. 1984. Scientific Explanation and the Causal Structure of the World. Princeton, N.J.: Princeton University Press.

Steiner, M. 1978. Mathematics, Explanation, and Scientific Knowledge. Noûs 12: 17-28.

Strevens, M. 2008. Depth: An Account of Scientific Explanation. Harvard, MA: Harvard University Press.

Strevens, M. 2013. No Understanding Without Explanation. Studies in History and Philosophy of Science Part A 44: 510-515.

Tóth, F. 1964. What the Bees Know and What They Do Not Know. Bull. Amer. Math. Soc. 70: 468-481.

Yoshimura, J. 1997. The Evolutionary Origins of Periodical Cicadas during Ice Ages. American Naturalist 149: 112-24.

Woodward, J. 2003. Making Things Happen: A Theory of Causal Explanation. New York: Oxford University Press.

GABRIEL TÂRZIU is an associate researcher in philosophy of mathematics at the Institute for Research in the Humanities, University of Bucharest. Currently, his research focuses on the problem of mathematical explanations of physical phenomena, and the role played by mathematics in the scientific understanding of the physical world.

AddRess: Institute for Research in the Humanities, University of Bucharest, St. Dimitrie Brândză, nr. 1, Bucharest, Romania. Email: gabi_tarziu@yahoo.com 